\title{
Grey Statistical Decision-making for Estimate of the Regional Wheat Trials Yield
}

\author{
Wuheng Gao* \\ School of Mathematical Sciences, University of Science and Technology of China, Hefei 230027, P. R. China \\ ${ }^{*}$ Corresponding author
}

\begin{abstract}
The regional wheat trials are used to objectively, fairly, and scientifically evaluate the yield and other characteristics of new wheat varieties grown in different wheat regions, and provide important scientific basis for the validation, ecological zoning, species distribution, and rational use of wheat varieties. It can accelerate the promotion of new wheat varieties. The evaluation of regional trial results is of great significance for further improving the scientificity and accuracy of the species trial. Grey statistical decision-making of grey system theory can fully weigh the trial results in different places and make a reasonable assessment of wheat varieties. In this paper, the method used to estimate the regional wheat trials yield is proposed, and 12 varieties of wheat varieties were evaluated with examples. The results were credible. Grey statistical Decisionmaking assessment method for the regional trial yield of wheat varieties proposed in this paper provides a practical method for the evaluation of regional wheat trial.
\end{abstract}

Keywords-wheat varieties; regional trial; grey statistical decision-making

\section{INTRODUCTION}

Regional wheat trials refer to conduct trials through uniform and standardized requirements in sites that can represent soil characteristics, climatic conditions, farming systems, and production levels in different natural areas of a unified ecological type area. Regional wheat trials can fully appraise the high yield, adaptability, stress resistance and quality of new varieties, and comprehensively evaluate the varieties based on the performance, resistance and quality. They are the scientific basis for evaluating varieties, and they are also important basis for the validation, promotion and scientific distribution of varieties [1, 2]. From selection to production, a wheat variety needs to go through a series of processes such as regional trials, production trials, validation, demonstration, and promotion, among which regional trials are particularly important.

At present, the commonly used methods for evaluating regional wheat trials include parameter estimation methods and non-parametric estimation methods [3,4]. The method of parameter estimation has evolved from the variance analysis to the combination of variance analysis and regression analysis popularized in the 1960s, and the combination of variance analysis and principal component analysis popularized in the 1980s, which has improved the efficiency of selecting varieties and their adaptation areas. Since the 1980s, researchers have begun to introduce non-parametric estimate method into the analysis of regional trial data. Rank analysis method is commonly used non-parametric estimation method [5, 6]. Jin Wenlin et al. (1999) put forward a rank analysis method to evaluate the yield traits of varieties under multiple environments. In rank analysis method, the grading value $\mathrm{H} 1$ of the variety under the same experimental environment is given according to the significance comparison result, and then is converted into the ranking value $\mathrm{H} 2$ in multiple environments, and these two parameters are used as one of the evaluation statistics [7, 8] of the yield performance. Through multiple comparisons of the significant differences in the rank averages among the varieties, the high yield of the varieties was evaluated and the influence of the experimental errors on the sorting of the varieties was reduced. The method proposed in this paper belongs to nonparametric estimation method.

The grey system theory was proposed by Professor Deng Julong, a Chinese scholar. It is an applied mathematics discipline with some clear information, some unclear information and uncertainties [9]. The grey theory holds that in the objective world, the abundantly presented system is not a white system (informatively explicit) nor a black system (informatively unclear), but a grey system. Since its inception, the grey system theory has been successfully applied in the fields of agriculture, industry, and meteorology.

Determining the action to be taken based on the actual situation and the predetermined goal is called decision-making. The grey decision-making is made in the case that the decisionmaking model contains grey elements or the general decision model is combined with the grey model. It focuses on the problem of program selection. That is, the selection of solutions under uncertain conditions. This depends to a large extent on the individual experience, attitude and determination of the decision makers. Grey statistical decision-making is applicable to the case of collective decision-making by multiple decision-making units. Its role is to integrate the opinions of various decision-making units to evaluate different decisionmaking schemes and make choices [10].

\section{STATISTICAL DECISION-MAKING ${ }^{[11]}$}

Suppose $a_{i}(i=1,2, \mathrm{~L}, n)$ is decision-making unit, $A=\left\{a_{1}, a_{2}, \mathrm{~L}, a_{n}\right\}$ is decision-making set, $b_{j}(j=1,2, \mathrm{~L}, n)$ is decision-making plan, $B=\left\{b_{1}, b_{2}, \mathrm{~L}, b_{m}\right\}$ is decision-making plan set, $\quad x_{i j}(i=1,2, \mathrm{~L}, n ; j=1,2, \mathrm{~L}, m) \quad$ is quantitative evaluation of $i$ decision-making unit for $j$ plan, 
$f^{k}(\mathrm{~g})(k=1,2, \mathrm{~L}, s)$ is whitening weight function of $k$ grey class. $\eta_{i}(i=1,2, \mathrm{~L}, n)$ is decision-making power of $i$ decision-making unit, $\sum_{i=1}^{n} \eta_{i}=1$, then

$$
\sigma_{j}^{k}=\frac{\sum_{i=1}^{n} f^{k}\left(x_{i j}\right) \cdot \eta_{i}}{\sum_{k=1}^{s} \sum_{i=1}^{n} f^{k}\left(x_{i j}\right) \cdot \eta_{i}},(j=1,2, \mathrm{~L}, m ; k=1,2, \mathrm{~L}, s)
$$

is called as decision-making coefficient of $j$ plan belonging to $k$ grey class.

And

$$
\sigma_{j}=\left(\sigma_{j}^{1}, \sigma_{j}{ }^{2}, \mathrm{~L}, \sigma_{j}^{s}\right) \quad, j=1,2, \mathrm{~L}, m
$$

is called as decision-making coefficient vector of $j$ plan.

If $\max _{1 \leq k \leq s}\left\{\sigma_{j}{ }^{k}\right\}=\sigma_{j}{ }^{* *}$, then $j$ plan is belong to $k^{*}$ grey class.

\section{The Determination OF the Whitening Weight FUNCTION}

According to the grey system theory, the commonly used whitening weight functions include three classes: lower class, intermediate class, and upper class. According to the figure, the segmented expression of whitening weight function of $i$ decision-making unit for $k$ class can be gotten [9].

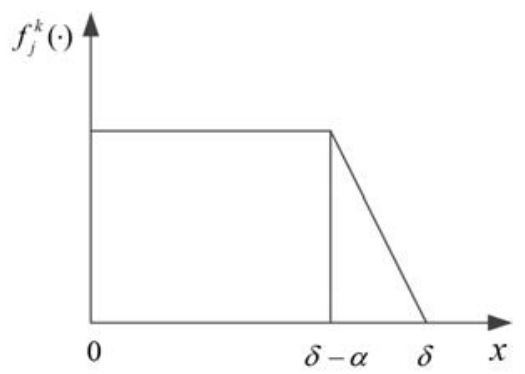

(A)

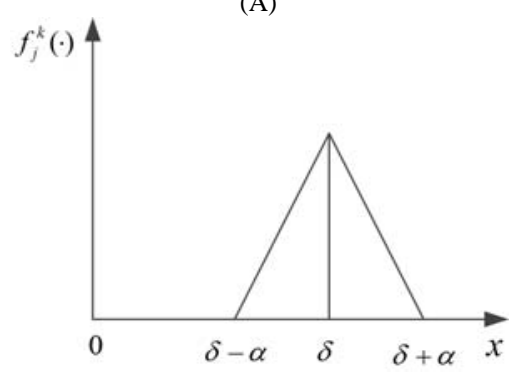

(B)

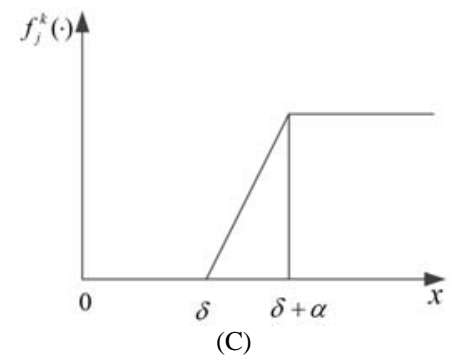

FIGURE I. WHITENING WEIGHT FUNCTION

(1) The lower class is shown in Figure I(A).

(2) The intermediate class is shown in Figure I(B).

(3) The upper class is shown in Figure I(C).

Where parameters $\delta$ and $\alpha$ are determined by experts.

\section{GREy STATISTICAL DECISION-MAKING FOR ESTIMATE OF THE REGIONAL WHEAT TRIALS YIELD}

Different wheat varieties are considered as different schemes, and the locations of different regions are considered as different evaluation units. The trials results in some locations show these species are good, and the trials results in other locations show other varieties are good. To integrate opinions from different locations, grey statistical decisions can be used for assessment the sort.

Not feasible grey class whitening weight function $f_{i}^{1}(x)$, feasible grey class whitening weight function $f_{i}^{2}(x)$ and excellent grey class whitening weight function $f_{i}^{3}(x)$ of regional wheat trials yield in different regions can be obtained by experts. Wheat yield in different regions can be put into $f_{i}^{1}(x), f_{i}^{2}(x), f_{i}^{3}(x)$ and be whitened. According to equation (1), grey statistical decision coefficient of different wheat varieties belong to three grey class $\sigma_{j}=\left(\sigma_{j}{ }^{1}, \sigma_{j}{ }^{2}, \sigma_{j}{ }^{3}\right)$ can be gotten. The wheat varieties belong to a certain grey class which the grey statistical decision coefficient is maxim.

\section{EXAMPLE}

The Huanghuai winter wheat region dryland group regional trials single-point yield in 2003-2004 is shown in Table I (data from the 2003-2004 national winter wheat dryland group trial summary) to assess the different varieties. If there are five varieties that can pass the certification, please assess which five varieties are.

After review by experts, Figure I(A) are selected as the infeasible grey class whitening weight function, Figure I(B) are selected as the feasible grey class whitening weight function, and Figure $\mathrm{I}(\mathrm{C})$ are selected as the optimal grey class whitening weight function. The average yield in different places is taken as $\delta$, and the ( highest output -lowest output)/2 in this place is taken as $\alpha$, shown in Table I. 
TABLE I. 2003-2004 HUANGHUAI WINTER WHEAT REGION DRYLAND GROUP REGIONAL TRIALS SINGLE-POINT YIELD

\begin{tabular}{|c|c|c|c|c|c|c|c|c|c|c|c|c|}
\hline & $\begin{array}{c}\text { Changwu } \\
\mathbf{5 8 - 6 - 4}\end{array}$ & $\begin{array}{c}\text { Hedong } \\
\mathbf{T X 0 6}\end{array}$ & $\begin{array}{c}\text { Yan } \\
\mathbf{9 6 2 6 6}\end{array}$ & $\begin{array}{c}\text { Han } \\
\mathbf{6 0 5 0}\end{array}$ & $\begin{array}{c}\text { Linkang } \\
\mathbf{1 1}\end{array}$ & $\begin{array}{c}\text { Lunong } \\
\mathbf{1 1 6}\end{array}$ & $\begin{array}{c}\text { Congmai } \\
\mathbf{1 3}\end{array}$ & $\begin{array}{c}\text { Yunhan } \\
\mathbf{2 2 - 3 3}\end{array}$ & $\begin{array}{c}\text { Kenong } \\
\mathbf{1 2 2}\end{array}$ & $\begin{array}{c}\text { Xiaobingmai } \\
\mathbf{1 1 3}\end{array}$ & $\begin{array}{c}\text { Zhoumai } \\
\mathbf{1 8}\end{array}$ & $\begin{array}{c}\text { Jinmai } \\
\mathbf{1 7}\end{array}$ \\
\hline Luoyang & 461.8 & 550.5 & 530.1 & $\mathbf{4 7 3 . 1}$ & 399.9 & 509.8 & 460.5 & 468.5 & 429.9 & 420.7 & 540.4 & 407.7 \\
\hline Hebi & 309.7 & 296.8 & 377.3 & 329.6 & 315.7 & 360.7 & 346.7 & 294.3 & 309.4 & 296.5 & 313.4 & 309.5 \\
\hline Songxian & 316.0 & 336.8 & 316.1 & 319.7 & 258.6 & 317.2 & 284.5 & 312.5 & 315.0 & 271.6 & 319.0 & 287.5 \\
\hline Sanmenxia & 213.9 & 256.9 & 222.2 & 223.6 & 229.2 & 230.6 & 251.4 & 263.9 & 180.6 & 254.2 & 227.8 & 226.4 \\
\hline Taian & 424.9 & 447.9 & 434.5 & 430.1 & 412.3 & 473.0 & 477.5 & 483.4 & 372.3 & 452.3 & 373.0 & 456.7 \\
\hline Zaozhuang & 406.7 & 397.5 & 393.2 & 388.3 & 348.3 & 377.5 & 369.2 & 363.3 & 341.4 & 335.0 & 359.2 & 325.8 \\
\hline Linyi & 410.0 & 418.8 & 416.6 & 395.1 & 386.7 & 401.2 & 383.0 & 420.9 & 362.0 & 385.4 & 394.1 & 370.4 \\
\hline Qingdao & 545.7 & 510.7 & 522.0 & 496.0 & 538.7 & 518.7 & 409.7 & 502.7 & 438.7 & 429.0 & 477.0 & 418.7 \\
\hline
\end{tabular}

TABLE II. $\delta$ AND $\alpha$ OF WHITENING WEIGHT FUNCTION

\begin{tabular}{|c|c|c|c|c|c|c|c|c|}
\hline & Luoyang & Hebi & Songxian & Sanmenxia & Taian & Zaozhuang & Linyi & Qingdao \\
\hline$\delta$ & 471.08 & 321.63 & 304.54 & 231.73 & 436.49 & 367.12 & 395.35 & 483.97 \\
\hline$\alpha$ & 75.30 & 41.50 & 39.10 & 41.65 & 55.55 & 40.45 & 29.45 & 68.00 \\
\hline$\delta-\alpha$ & 395.78 & 280.13 & 265.44 & 190.08 & 380.94 & 326.67 & 365.9 & 415.97 \\
\hline$\delta+\alpha$ & 546.38 & 363.13 & 343.64 & 273.38 & 492.04 & 407.57 & 424.8 & 551.97 \\
\hline
\end{tabular}

According to the figure, the subsection expressions of the grey class whitening weight function in Luoyang can be written.

(Luoyang)Not feasible grey class can be given by

$$
f_{1}^{1}(x)=\left\{\begin{array}{cc}
0, & x<0 \\
1, & 0 \leq x \leq 395.78 \\
\frac{-x+471.08}{75.30}, & 395.78<x \leq 471.08 \\
0 & x>471.08
\end{array}\right.
$$

(Luoyang)Feasible grey class can be given by

$$
f_{1}^{2}(x)=\left\{\begin{array}{cc}
0, & x<395.78 \\
\frac{x-395.78}{75.30}, & 395.78 \leq x \leq 471.08 \\
\frac{-x+546.38}{75.30}, & 471.08<x \leq 546.38 \\
0, & x>546.38
\end{array}\right.
$$

(Luoyang) Excellent grey class can be given by

$$
f_{1}^{3}(x)=\left\{\begin{array}{cc}
0, & x<471.08 \\
\frac{x-471.08}{75.30}, & 471.08 \leq x \leq 546.38 \\
1, & x>546.38
\end{array}\right.
$$

The subsection expressions of the grey class whitening weight functions in other places will not listed one by one. In general, the subsection expressions of infeasible grey class, feasible grey class, and excellent grey class in different places are

Infeasible grey class can be given by

$$
f_{i}^{1}(x)=\left\{\begin{array}{cc}
0, & x<0 \\
1, & 0 \leq x \leq(\delta-\alpha) \\
\frac{-x+\delta}{\alpha}, & (\delta-\alpha)<x \leq \delta \\
0 & x>\delta
\end{array}\right.
$$

Feasible grey class can be given by

$$
f_{i}^{2}(x)=\left\{\begin{array}{cc}
0, & x<(\delta-\alpha) \\
\frac{x-(\delta-\alpha)}{\alpha}, & (\delta-\alpha) \leq x \leq \delta \\
\frac{-x+(\delta+\alpha)}{\alpha}, & \delta<x \leq(\delta+\alpha) \\
0, & x>(\delta+\alpha)
\end{array}\right.
$$

Excellent grey class can be given by 


$$
f_{1}^{3}(x)=\left\{\begin{array}{cc}
0, & x<\delta \\
\frac{x-\delta}{\alpha}, & \delta \leq x \leq(\delta+\alpha) \\
1, & x>(\delta+\alpha)
\end{array}\right.
$$

The experts consider that the evaluation of each place is very important, and the decision-making power in each place is

$$
\eta_{1}=\eta_{2}=\mathrm{L}=\eta_{12}=1 / 12
$$

Thus, the grey statistical decision coefficient for all varieties belonging to infeasible grey class, feasible grey class and excellent grey class can be obtained.

$$
\begin{aligned}
& \sigma_{1}=\left(\sigma_{1}^{1}, \sigma_{1}^{2}, \sigma_{1}^{3}\right)=(0.1309, \quad 0.5345, \quad 0.3346) \\
& \sigma_{2}=\left(\sigma_{2}^{1}, \sigma_{2}^{2}, \sigma_{2}^{3}\right)=(0.0748, \quad 0.3533, \quad 0.5719) \\
& \sigma_{3}=\left(\sigma_{3}^{1}, \sigma_{3}^{2}, \sigma_{3}^{3}\right)=(0.0331, \quad 0.4663, \quad 0.5006) \\
& \sigma_{4}=\left(\sigma_{4}^{1}, \sigma_{4}^{2}, \sigma_{4}^{3}\right)=(0.0398, \quad 0.7968, \quad 0.1634) \\
& \sigma_{5}=\left(\sigma_{5}^{1}, \sigma_{5}^{2}, \sigma_{5}^{3}\right)=(0.4179, \quad 0.4815, \quad 0.1006) \\
& \sigma_{6}=\left(\sigma_{6}^{1}, \sigma_{6}^{2}, \sigma_{6}^{3}\right)=(0.0034, \quad 0.5713, \quad 0.4253) \\
& \sigma_{7}=\left(\sigma_{7}^{1}, \sigma_{7}^{2}, \sigma_{7}^{3}\right)=(0.2590, \quad 0.5077, \quad 0.2333) \\
& \sigma_{8}=\left(\sigma_{8}^{1}, \sigma_{8}^{2}, \sigma_{8}^{3}\right)=\left(\begin{array}{lll}
0.0984, & 0.5312, & 0.3704
\end{array}\right) \\
& \sigma_{9}=\left(\sigma_{9}^{1}, \sigma_{9}^{2}, \sigma_{9}^{3}\right)=\left(\begin{array}{lll}
0.6429, & 0.3237, & 0.0334
\end{array}\right) \\
& \sigma_{10}=\left(\sigma_{10}^{1}, \sigma_{10}^{2}, \sigma_{10}^{3}\right)=(0.5072, \quad 0.3898, \quad 0.1030) \\
& \sigma_{11}=\left(\sigma_{11}^{1}, \sigma_{11}^{2}, \sigma_{11}^{3}\right)=(0.2042, \quad 0.6345, \quad 0.1613) \\
& \sigma_{12}=\left(\sigma_{12}^{1}, \sigma_{12}^{2}, \sigma_{12}^{3}\right)=(0.5631,0.3914, \quad 0.0455)
\end{aligned}
$$

From

$$
\begin{aligned}
& \max _{1 \leq k \leq 3}\left\{\sigma_{1}^{k}\right\}=0.5345=\sigma_{1}^{2} \max _{1 \leq k \leq 3}\left\{\sigma_{2}^{k}\right\}=0.5719=\sigma_{2}^{3}, \\
& \max _{1 \leq k \leq 3}\left\{\sigma_{3}^{k}\right\}=0.5006=\sigma_{3}^{3} \max _{1 \leq k \leq 3}\left\{\sigma_{4}^{k}\right\}=0.7968=\sigma_{4}^{2}, \\
& \max _{1 \leq k \leq 3}\left\{\sigma_{5}^{k}\right\}=0.4815=\sigma_{5}^{2}, \max _{1 \leq k \leq 3}\left\{\sigma_{6}^{k}\right\}=0.5713=\sigma_{6}^{2}, \\
& \max _{1 \leq k \leq 3}\left\{\sigma_{7}^{k}\right\}=0.5077=\sigma_{7}^{2} \max _{1 \leq k \leq 3}\left\{\sigma_{8}^{k}\right\}=0.5312=\sigma_{8}^{2}, \\
& \max _{1 \leq k \leq 3}\left\{\sigma_{9}^{k}\right\}=0.6429=\sigma_{9}^{1}, \max _{1 \leq k \leq 3}\left\{\sigma_{10}^{k}\right\}=0.5072=\sigma_{10}^{1}, \\
& \max _{1 \leq k \leq 3}\left\{\sigma_{11}^{k}\right\}=0.6345=\sigma_{11}^{2}, \max _{1 \leq k \leq 3}\left\{\sigma_{12}^{k}\right\}=0.5631=\sigma_{12}^{1},
\end{aligned}
$$

it can be seen that, varieties 2 and 3 belong to the excellent grey class, and varieties $1,4,5,6,7,8$, and 11 belong to the feasible grey class, and varieties 9,10 , and 12 belong to the infeasible grey class.

Comparing varieties 1 and 2, variety 2 is slightly better (Possibility of being excellent gray class is big).

When comparing the varieties $1,4,5,6,7,8$, and 11 , the order of the varieties is: varieties $6,4,8,11,11,7$, and 5 .

Comparing varieties 9, 10 and12, variety 10 is slightly better (Possibility of being infeasible gray class is small). Then the order is 12,9 .

If there are five varieties that can pass the validation, it is recommended that varieties $2,3,4,5$, and 8 pass the validation.

\section{CONCLUSION}

Grey statistical decision-making is used in the case of collective decision making by multiple decision units. Here different decision units are different places, and the performances of different varieties in different places are not the same, but the results of comprehensive comparison can still see the superiority or inferior of different varieties.

The analysis results of this paper are only for the analysis of more places of one year. For more places of two years and more places of three years of experiments, more research of comprehensive evaluation for regional trial results of different years is needed.

\section{REFERENCES}

[1] Haihua W, Yuwen L, Mingkai CH, Zhanbin W. How to do wheat regional trials [J]. Agricultural Science and Technology News, 2016(02): 5-7.

[2] Gang W, Wenjun D, Jincang L. Problems and countermeasures of the current regional wheat experiment in dryland [J]. Shaanxi Journal of Agricultural Sciences, 2015, 61(11): 77-78+99.

[3] Shaohua S. How to make a regional evaluation of wheat trials [J]. Chinese Seed Industry, 2016(03): 24-25.

[4] Lei CH, Fanxiang H, Shoujun CH, Yun Y, Delong Y, Changgang Y, Caixia $\mathrm{H}$, Hongbo $\mathrm{CH}$. Accuracy of regional wheat experiment in China and its comprehensive evaluation [J]. Chinese Journal of Applied Ecology, 2013, 24(10): 2814- 2820.

[5] Simei ZH, Sijun Y, Kejun G, Henggan ZH, Bo X, Zhe CH. Yield characteristics and stability analysis of wheat in regional trials [J]. Chinese Agricultural Science Bulletin, 2012, 28(03): 172-176.

[6] Xiaoli N, Tao J, Qing W, Wei W, Falong J, Jianfeng L, Zhong R. Study on evaluation of yield characters of peanut in anhui province by rank analysis [J]. Chinese Agricultural Science Bulletin, 2017, 33(01): 18- 23.

[7] Ouslimani, F., Ameur, Z., Ouslimani, A.. Texture analysis method using regional rank coding[P]. Communication Systems, Networks \& Digita Signal Processing (CSNDSP), 2014 9th International Symposium on, 2014.

[8] Gluchowski, P., Kucharski, A.A.. Comparison of the low rank IE-QR algorithm and the classical method of moments in the analysis of layered media problems[P]. Microwave Radar and Wireless Communications (MIKON), 2012 19th International Conference on, 2012.

[9] Julong D. Theory foundation of grey [M]. Wuhan: Huazhong University of Science and Technology Press. 2002

[10] Julong D. Grey prediction and grey decision [M]. Wuhan: Huazhong University of Science and Technology Press. 2002

[11] Sifeng L, et al. Theory of grey system and its application [M]. Beijing: Science Press, 2018 